KUNS 1180

\title{
Momentum distribution of fragments in heavy ion reactions: Dependence on the stochastic collision process
}

\author{
Akira Ono, Hisashi Horiuchi and Toshiki Maruyama \\ Department of Physics, Kyoto University, Kyoto 606-01, Japan \\ Akira Ohnishi \\ Research Center for Nuclear Physics, Osaka University, Ibaraki 567, Japan
}

(January 21, 1993)

\begin{abstract}
Momentum distribution of fragments produced in ${ }^{12} \mathrm{C}+{ }^{12} \mathrm{C}$ reaction at 28.7 $\mathrm{MeV} /$ nucleon is analyzed with antisymmetrized version of molecular dynamics (AMD). Calculations are made for several cases of stochastic collisions and the projectile fragmentation peak in momentum distribution is reproduced by the incorporation of many-body nature in stochastic collision process. Furthermore the value of the cross section is found to be reflected in the low momentum part of fragments such as alpha particles and ${ }^{9} \mathrm{Be}$.
\end{abstract}




\section{INTRODUCTION}

Fragment formation is the most characteristic feature of the heavy ion reactions in the intermediate energy region. The main reaction mechanism of the peripheral collision is the projectile fragmentation, in which a projectile-like fragment produced by stripping some nucleons from the projectile is emitted with the velocity close to the beam velocity. In the central collision, on the other hand, the reaction mechanism is more complicated and many fragments with various mass numbers are produced. At the same time, if we focus on the collective momentum flow instead of fragment mass number, the central collision in this energy region is believed to be a good tool to investigate the equation of state of nuclear matter.

There are many models which describe these features of intermediate energy heavy ion reactions. Abrasion-ablation model, for example, gives a very intuitive picture of projectile fragmentation and is successful in reproducing the mass distribution of projectile-like fragments. This model, however, assumes the reaction mechanism itself, and cannot answer the question whether the projectile fragmentation is possible in low energy heavy ion reactions. In these years, projectile fragmentation is observed in experiments at the incident energies down to $30 \mathrm{MeV} /$ nucleon. It may not be trivial theoretically that participant spectator picture is true at this incident energy comparable to the Fermi energy of nuclei. The central collisions, on the other hand, are sometimes described by the statistical models if one put emphasis on the multifragmentation as the phase transition. In the statistical models, however, dynamical effects are not fully incorporated and collective momentum flow is usually calculated by VUU approach which cannot describe fragment formation without further assumptions.

In order to study these phenomena in a unified way with few assumptions on reaction mechanisms, microscopic simulations which can treat fragment formation is indispensable. Quantum molecular dynamics (QMD) $[1,2]$ has been the most powerful among such microscopic simulation methods. QMD is, however, largely of classical nature and, for example, it cannot describe shell effects neither of colliding individual nuclei nor in the reaction process. In the previous work $[3,4]$, we constructed an antisymmetrized version of molecular dynamics (AMD) by incorporating two-nucleon collision process as the residual interaction into the fermionic molecular dynamics (FMD) [5] proposed by Feldmeier. AMD describes the system with a Slater determinant of Gaussian wave packets, and therefore can describe quantum mechanical features such as shell effects. Furthermore AMD can describe fragment formation as well as QMD. These features of AMD has been demonstrated by the calculation of mass distribution of fragments in the previous work [3,4] with the combination to the statistical cascade decay calculation. AMD can also be applied to the study of nuclear structure of light nuclei $[6,7]$ with some extensions of the wave function.

In this paper, the momentum distribution of fragments produced in ${ }^{12} \mathrm{C}+{ }^{12} \mathrm{C}$ reaction at $28.7 \mathrm{MeV} /$ nucleon is mainly discussed because it has much more information on the reaction mechanisms such as projectile fragmentation than the mass distribution does. Since the incorporation of the stochastic collision process has some ambiguity, we have made calculations with different types of stochastic collision process, not only by changing the cross section but also by including many-body nature such as nucleon-alpha collisions in addition to the usual two-nucleon collisions. It will turn out that the momentum distribution 
of fragments is very sensitive to the stochastic collision process. This fact means that detailed analysis of momentum distribution of fragments is powerful to settle the ambiguity of stochastic collision process which has been the main obstacle in extracting the equation of state of nuclear matter by the analysis of collective momentum flow.

In Sec. II, the framework of AMD, with the inclusion nucleon-alpha collisions in addition to the usual two-nucleon collisions, is presented. In Sec. III, the results of calculation for the reaction ${ }^{12} \mathrm{C}+{ }^{12} \mathrm{C}$ are compared to the data, and the dependence of the momentum distribution of fragments on the stochastic collision process is discussed, which will reveal the reaction mechanisms both of the peripheral collision and of the central collision. The manifestation of collective momentum flow of fragments will also be mentioned. Finally in Sec. IV, we will summarize the results and discuss future problems.

\section{FORMULATION OF AMD}

Since the framework of the antisymmetrized version of molecular dynamics (AMD) was described in detail in Ref. [4], here is shown only the outline of our framework about the wave function and the equation of motion. The treatment of the stochastic collision process is updated from Ref. [4], and therefore is described here in detail.

\section{A. Wave function}

In AMD, the wave function of $A$-nucleon system $|\Phi\rangle$ is described by a Slater determinant $|\Phi(\mathbf{Z})\rangle$

$$
|\Phi(\mathbf{Z})\rangle=\frac{1}{\sqrt{A !}} \operatorname{det}\left[\varphi_{i}(j)\right], \quad \varphi_{i}=\phi_{\mathbf{Z}_{i}} \chi_{\alpha_{i}}
$$

where $\alpha_{i}$ represents the spin-isospin label of $i$-th single particle state, $\alpha_{i}=\mathrm{p} \uparrow, \mathrm{p} \downarrow, \mathrm{n} \uparrow$, or $\mathrm{n} \downarrow$, and $\chi$ is the spin-isospin wave function. $\phi_{\mathbf{Z}_{i}}$ is the spatial wave function of $i$-th single particle state which is a Gaussian wave packet

$$
\left\langle\mathbf{r} \mid \phi_{\mathbf{Z}_{i}}\right\rangle=\left(\frac{2 \nu}{\pi}\right)^{3 / 4} \exp \left[-\nu\left(\mathbf{r}-\frac{\mathbf{Z}_{i}}{\sqrt{\nu}}\right)^{2}+\frac{1}{2} \mathbf{Z}_{i}^{2}\right]
$$

where the width parameter $\nu$ is treated as time-independent in our model. We took $\nu=0.16$ $\mathrm{fm}^{-2}$ in the calculation presented in this paper. If we define $\mathbf{D}$ and $\mathbf{K}$ as $\mathbf{Z}=\sqrt{\nu} \mathbf{D}+$ $(i / 2 \hbar \sqrt{\nu}) \mathbf{K}$, then

$$
\begin{aligned}
\left\langle\phi_{\mathbf{Z}}|\mathbf{r}| \phi_{\mathbf{Z}}\right\rangle /\left\langle\phi_{\mathbf{Z}} \mid \phi_{\mathbf{Z}}\right\rangle & =\mathbf{D}, \\
\left\langle\phi_{\mathbf{Z}}|\mathbf{p}| \phi_{\mathbf{Z}}\right\rangle /\left\langle\phi_{\mathbf{Z}} \mid \phi_{\mathbf{Z}}\right\rangle & =\mathbf{K} .
\end{aligned}
$$




\section{B. Equation of motion}

The time developments of the centers of Gaussians, $\left\{\mathbf{Z}_{i}(i=1,2, \ldots, A)\right\}$, are determined by two processes. One is the time development determined by the time-dependent variational principle

$$
\delta \int_{t_{1}}^{t_{2}} d t \frac{\left\langle\Phi(\mathbf{Z})\left|\left(i \hbar \frac{d}{d t}-H\right)\right| \Phi(\mathbf{Z})\right\rangle}{\langle\Phi(\mathbf{Z}) \mid \Phi(\mathbf{Z})\rangle}=0
$$

which leads to the equation of motion for $\{\mathbf{Z}\}$;

$$
i \hbar \sum_{j \tau} C_{i \sigma, j \tau} \dot{Z}_{j \tau}=\frac{\partial \mathcal{H}}{\partial Z_{i \sigma}^{*}} \quad \text { and c.c. }
$$

where $\sigma, \tau=x, y, z . \mathcal{H}$ is the expectation value $\langle H\rangle$ of quantum mechanical Hamiltonian $H$,

$$
\mathcal{H}\left(\mathbf{Z}, \mathbf{Z}^{*}\right)=\frac{\langle\Phi(\mathbf{Z})|H| \Phi(\mathbf{Z})\rangle}{\langle\Phi(\mathbf{Z}) \mid \Phi(\mathbf{Z})\rangle}
$$

and

$$
C_{i \sigma, j \tau}=\frac{\partial^{2}}{\partial Z_{i \sigma}^{*} \partial Z_{j \tau}} \log \langle\Phi(\mathbf{Z}) \mid \Phi(\mathbf{Z})\rangle
$$

is a positive definite hermitian matrix.

The same effective interaction as in the previous work [3,4], i.e., Volkov No.1 [8] with $m=$ 0.576 is used. Coulomb interaction is included. The problem of the zero-point oscillation of the fragment center-of-mass motion is overcome phenomenologically with the prescription we have proposed in Ref. [4]. As has been shown in Ref. [4], the binding energies of nuclei lighter than ${ }^{12} \mathrm{C}$ are reproduced very well by this choice of parameters concerned with equation of motion.

\section{Stochastic collision process}

The second process which determines the time development of the system is the stochastic collision process due to the residual interaction. We incorporate this process in the similar way to QMD, i.e., the momenta of two nucleons which have approached toward each other are changed stochastically. In AMD, however, this is not straightforward to do since the centers of Gaussian wave packets $\left\{\mathbf{Z}_{i}\right\}$ are no more physical coordinates of nucleons due to the effect of antisymmetrization. We overcome this difficulty by introducing the physical coordinates $\left\{\mathbf{W}_{i}\right\}[3,4]$ as

$$
\mathbf{W}_{i}=\sum_{j=1}^{A}(\sqrt{Q})_{i j} \mathbf{Z}_{j}
$$

where 


$$
Q_{i j}=\frac{\partial}{\partial\left(\mathbf{Z}_{i}^{*} \cdot \mathbf{Z}_{j}\right)} \log \langle\Phi(\mathbf{Z}) \mid \Phi(\mathbf{Z})\rangle .
$$

The position $\mathbf{R}_{j}$ and momentum $\mathbf{P}_{j}$ of $\mathbf{W}_{j}=\sqrt{\nu} \mathbf{R}_{j}+(i / 2 \hbar \sqrt{\nu}) \mathbf{P}_{j}$ can be interpreted to be physical position and momentum coordinates of nucleons [4].

As a manifestation of Pauli principle, there is Pauli-forbidden region in the phase space of physical coordinates. Taking account of this fact, the Pauli-blocking in the final state of two-nucleon collision is introduced in a natural way [3,4], i.e., the collision is Pauli-blocked if the physical coordinate of the final state is in the Pauli-forbidden region.

The in-medium cross section of two-nucleon collision is parametrized as

$$
\sigma_{N N}=\frac{100 \mathrm{mb}}{1+E / 200 \mathrm{MeV}+C \min \left(\left(\rho / \rho_{0}\right)^{2}, 1\right)},
$$

where $E$ is the energy in the laboratory system of two-nucleon scattering, and $\rho$ is the density at the middle point of colliding two nucleons. The parameter $C$ controls the reduction of the cross section due to the medium effect. Isotropic scattering is assumed.

So far we considered only two-nucleon collisions as the effect of residual interaction. There may be, however, other effects of residual interaction in which more than two nucleons are concerned. A simple example which shows the necessity of the many-body nature of residual interaction in the AMD framework is the proton-alpha elastic scattering which is the dominant process in $p+\alpha$ reaction up to the intermediate energy region. First of all, the incident proton is deflected at most $20^{\circ}$ by the equation of motion of AMD if the incident energy is greater than $30 \mathrm{MeV}$, though the large angle elastic scattering is observed in experiments. The two-nucleon collision process in AMD does not remedy this situation because the alpha particle is inevitably excited once the two-nucleon collision occurs between the incident proton and a nucleon in the alpha particle.

In order to study the effect of many-body nature of residual interaction in heavy ion reactions, we also incorporate following many-body stochastic collisions, which we call nucleonalpha collisions, in addition to the usual two-nucleon collisions. When a nucleon and an alpha cluster (a cluster of four physical coordinates with different spin-isospins, which is judged by the chain clustering method with the critical distance $|\Delta \mathbf{W}|=0.25$ ) have approached toward each other, (i) they are scattered elastically with the cross section $\sigma_{N \alpha, \mathrm{el}}(E, \rho)$ and (ii) the nucleon and a nucleon in the alpha cluster are scattered in the usual way with the cross section $\sigma_{N \alpha \text {,inel }}(E)$. The same energy and density dependence as that of the two-nucleon collision is assumed for the in-medium total nucleon-alpha cross section as

$$
\begin{aligned}
\sigma_{N \alpha, \text { tot }}(E, \rho) & =\sigma_{N \alpha, \mathrm{ll}}(E, \rho)+\sigma_{N \alpha, \text { inel }}(E) \\
& =\frac{571 \mathrm{mb}}{1+E / 200 \mathrm{MeV}+C \min \left(\left(\rho / \rho_{0}\right)^{2}, 1\right)}
\end{aligned}
$$

As for the energy dependent inelastic cross section, we use the experimental data of reaction cross section [9] (see Fig. 1) which can be parametrized as

$$
\sigma_{N \alpha, \text { inel }}(E)=\max \left(120-162 e^{-(E-20 \mathrm{MeV}) / 10 \mathrm{MeV}}, 0\right) \mathrm{mb} .
$$




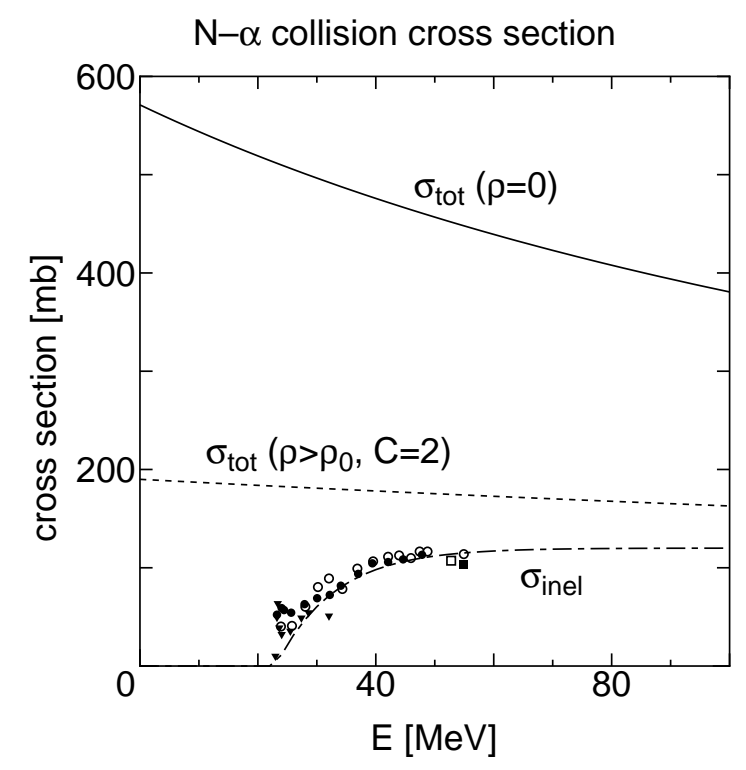

FIG. 1. Energy dependence of the cross sections of nucleon-alpha collisions. Solid line and dotted line are the total cross sections parametrized as Eq. (12), for $\rho=0$ and $\rho \geq \rho_{0}(C=2)$ respectively. Dot dashed line is the inelastic cross section parametrized as Eq. (13) and various symbols are the experimental data of reaction cross section of $p+\alpha$ reaction presented in Ref. [9].

The angular distribution of the elastic nucleon-alpha scattering is assumed to be

$$
\frac{d \sigma_{N \alpha, \mathrm{el}}}{d \Omega} \propto \exp \left[-\left(\frac{180^{\circ}-\theta}{70^{\circ}}\right)^{2}\right]+10 \exp \left[-\left(\frac{\theta-20^{\circ}}{40^{\circ}}\right)^{2}\right]
$$

The parametrization of Eqs. (12), (13) and (14) reproduces the data of elastic differential cross section of $p+\alpha$ reaction at $E=28.1 \mathrm{MeV}[9]$ for $\theta \geq 30^{\circ}$ in the case $\rho=0$ as shown in Fig. 2. Note that it is not necessary to reproduce the data for small scattering angles since the small angle elastic scatterings are described not only by the stochastic collisions but also by the equation of motion of AMD. The energy dependences of total and inelastic cross sections are shown in Fig. 1, where the data of the reaction cross section are also shown. The parametrization is consistent with the essential feature that the elastic cross section decreases as the energy increases.

When the alpha cluster composed of nucleons $i_{1}, i_{2}, i_{3}$ and $i_{4}$ is to be scattered elastically, the momentum transfer which should be received by the alpha cluster is shared not only by the four nucleons but also by the other nucleons $j$ with the weights proportional to $\exp \left(-4\left|\mathbf{Z}_{j}-\mathbf{Z}_{i_{\alpha}}\right|^{2}\right)$, where the nucleons $j$ and $i_{\alpha}$ have the same spin and isospin. This prescription is aimed to suppress the Pauli-blocking probability of the scattered alpha cluster which seems too large but for this prescription. When two alpha clusters have approached, nucleon-alpha collisions defined above are made between nucleons in one alpha cluster and the other alpha cluster. It is randomly decided which alpha cluster is treated as merely four nucleons.

Although a procedure to scatter two particles with the given cross section $\sigma$ has been 


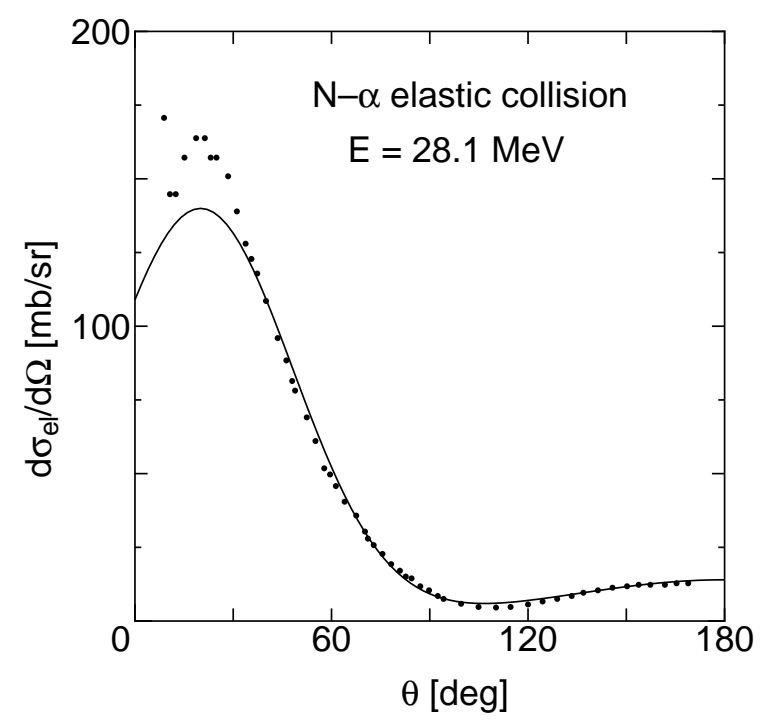

FIG. 2. Differential cross section of nucleon-alpha elastic collisions at $E=28.1 \mathrm{MeV}$. Solid line shows the parametrization of Eq. (14) and dots are the data [9].

described in Ref. [4], the scattering probability appearing in this procedure exceeds unity when it is applied to the nucleon-alpha collisions. In the calculation presented in this paper, we use the following procedure when nucleon-alpha collision process is included. At each time step, it is examined whether each pair of two particles (which may be two nucleons or a nucleon and an alpha cluster) should make a stochastic collision. The probability with which the two particles are scattered (up to the Pauli-blocking) when the relative coordinate changes from $\mathbf{r}$ to $\mathbf{r}+d \mathbf{r}$ in a time interval $d t$ is assumed to be proportional to the density overlap of the two Gaussian wave packets as

$$
P(\mathbf{r})|d \mathbf{r}|=\alpha e^{-\nu \mathbf{r}^{2}}|d \mathbf{r}|,
$$

where the proportionality constant $\alpha$ is determined by the condition that the total cross section should be $\sigma$ if two particles move on straight lines before they are scattered,

$$
2 \pi \int b d b\left[1-\exp \left(-\alpha \sqrt{\pi / \nu} e^{-\nu b^{2}}\right)\right]=\sigma .
$$

This condition can be rewritten as

$$
y=-\sum_{n=1}^{\infty} \frac{(-x)^{n}}{n \cdot n !},
$$

where

$$
y=\frac{\nu \sigma}{\pi}, \quad x=\alpha \sqrt{\frac{\pi}{\nu}}
$$

When more than two stochastic collisions should occur at the same time step, the order of collisions is decided randomly. 


\section{RESULTS AND DISCUSSIONS}

\section{A. Numerical calculation}

AMD simulations are made for the reaction ${ }^{12} \mathrm{C}+{ }^{12} \mathrm{C}$ at the incident energy 28.7 $\mathrm{MeV} /$ nucleon. The initial state of each simulation is constructed by boosting randomly rotated two ${ }^{12} \mathrm{C}$ nuclei in ground states which have been obtained by frictional cooling method [6,3,4]. Simulations are repeated for many times (typically 1000 times) for various impact parameters and for different random seeds, and each simulation is considered to correspond to an experimental event.

AMD calculations are made for three types of stochastic collision process. The calculation presented in Refs. [3,4], where only the two-nucleon collisions are incorporated and the parameter $C$ in Eq. (11) which controls the medium effect is taken to be 0, is called the case (a) here. In the cases (b) and (c), both the two-nucleon collisions and nucleon-alpha collisions are incorporated, and $C=1$ for the case (b), and $C=2$ for the case (c). Note that the cross section is smaller for larger value of $C$.

After the AMD calculation which is truncated at $t \approx 200 \mathrm{fm} / c$, most of the produced fragments are in their excited states and should decay into lighter fragments before they are detected in experiment. We calculate statistical cascade decay of each of these fragments by using a code $[10,3,4]$ which is similar to CASCADE of Pühlhofer [11].

\section{B. Mass distribution}

The calculated isotope distribution of fragments is compared to the experimental data in Fig. 3 for the case (c). As in the case (a) which we have already discussed in Refs. [3,4], the mass distribution is reproduced very well, especially the large production cross section of alpha particles. From Fig. 4 in which mass distributions for two stochastic collision processes (b) and (c) are compared, we can see that the mass distribution of fragments lighter than the projectile and the target is insensitive to the stochastic collision process. The cross sections of heavier fragments are, on the other hand, reflect the stochastic collision cross section. The smaller the parameter $C$ is, i.e., the larger the cross section of stochastic collisions is, the more abundantly heavy fragments are produced.

\section{Projectile fragmentation}

In Fig. 5, the energy spectra of ${ }^{9} \mathrm{Be}$ at the angle $5.5^{\circ}$ are compared to the experimental data [12] for three cases of stochastic collisions. In experiment, the projectile fragmentation peak appears very close to the beam velocity even in this relatively low incident energy $E=28.7 \mathrm{MeV} /$ nucleon, but the calculation (a) only with two-nucleon collisions fails to explain this phenomenon. This failure can be understood by the fact that the momentum of the nucleon in the projectile which has experienced a two-nucleon collision with a nucleon in the target is too small in the projectile frame for the nucleon to escape from the projectile. If two nucleons which make a collision have zero momenta in the projectile and the target frame respectively and the scattering angle is $90^{\circ}$, which is the most probable case, the 


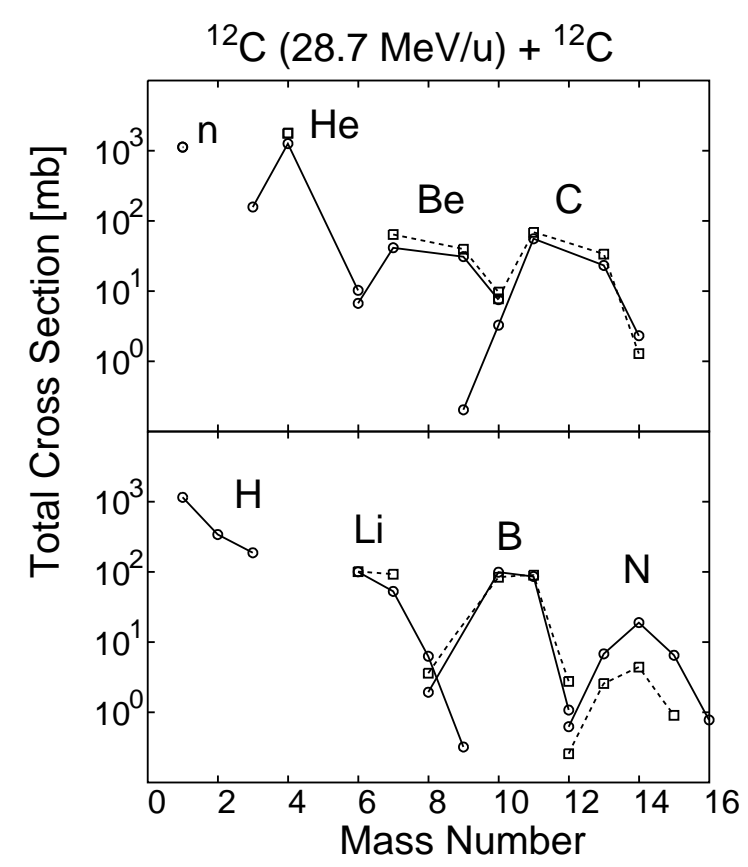

FIG. 3. Isotope distribution of produced fragments. The results of calculation in the case (c) are shown by circles connected by solid lines, and the data are shown by squares connected by dotted lines.

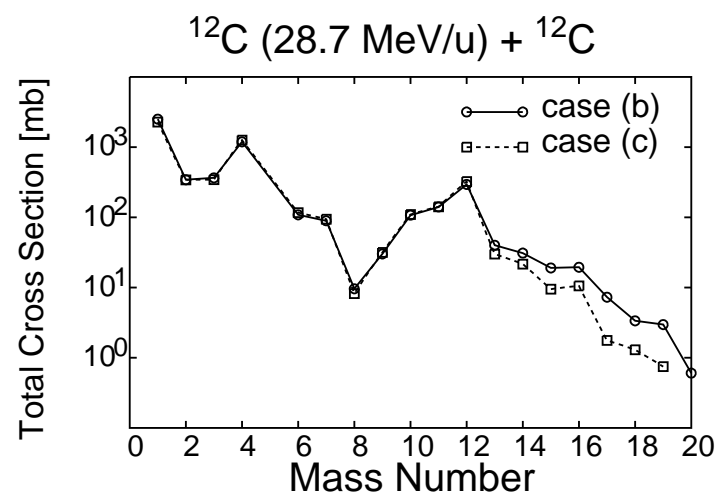

FIG. 4. Calculated mass distribution for the cases (b) and (c) of stochastic collision process. 


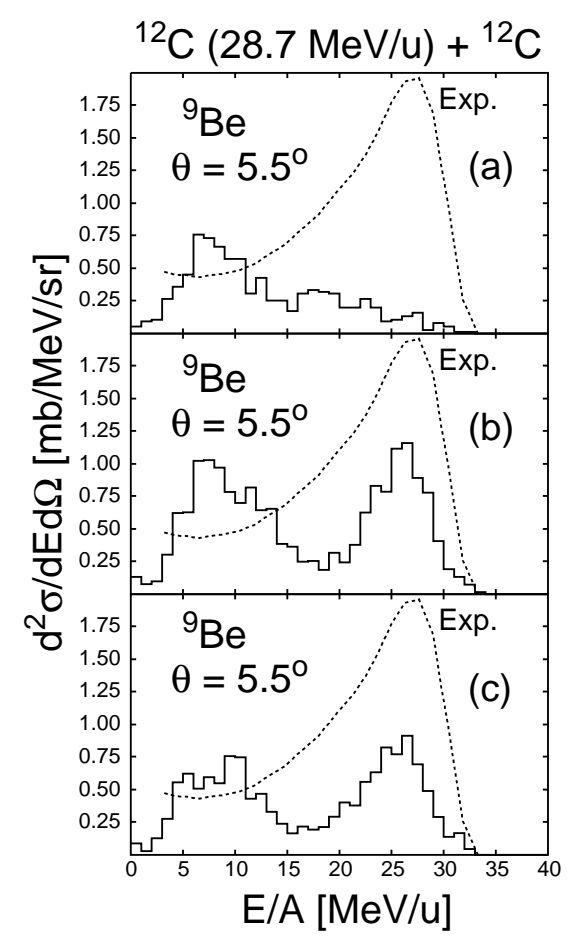

FIG. 5. Energy spectra of ${ }^{9}$ Be at $\theta=5.5^{\circ}$ for three cases (a), (b) and (c) of stochastic collision process. Histograms are the result of calculation and the dotted curves are the data.

nucleon in the projectile has the kinetic energy $E / 2$ in the projectile frame (as long as the energy correction [4] in the two-nucleon collision is ignored), where $E$ is the incident energy per nucleon in the laboratory system. Since $E=28.7 \mathrm{MeV} /$ nucleon in the present case, the typical kinetic energy of scattered nucleon in the projectile is $15 \mathrm{MeV}$. It is difficult for this scattered nucleon to escape from the projectile since the proton separation energy of ${ }^{12} \mathrm{C}$ is also about $15 \mathrm{MeV}$.

This situation evidently depends on the incident energy. In fact, in the calculation with the incident energy $70 \mathrm{MeV} /$ nucleon and the impact parameter $6 \mathrm{fm}$, and only with twonucleon collisions, we have found that 15 projectile-like and target-like fragments with mass number 11, 10 and 9 have been produced in 58 events before statistical cascade decay, while only one projectile-like fragment is found in the same calculation but with the incident energy $28.7 \mathrm{MeV} /$ nucleon.

The inclusion of many-body nature in stochastic collision process has the effect to increase the kinetic energy of scattered nucleon in the projectile, and therefore the nucleon can easily escape from the projectile or, otherwise, the excitation energy of the projectile is large enough for the projectile to decay into ${ }^{9} \mathrm{Be}$. By the inclusion of nucleon-alpha collisions, we have succeeded in the reproduction of projectile fragmentation peak in energy spectrum (Fig. 5), at least qualitatively. We have checked that the calculated projectile fragmentation peak comes from the impact parameter range $b>5 \mathrm{fm}$ as shown in Fig. 6, and in Fig. 7 the effect of statistical cascade decay on the energy spectrum of ${ }^{9} \mathrm{Be}$ is shown. It is seen that most of the projectile-like ${ }^{9} \mathrm{Be}$ fragments are not produced directly in AMD calculation but they are the decay products of excited fragments with mass numbers 10, 11 and 12 . 


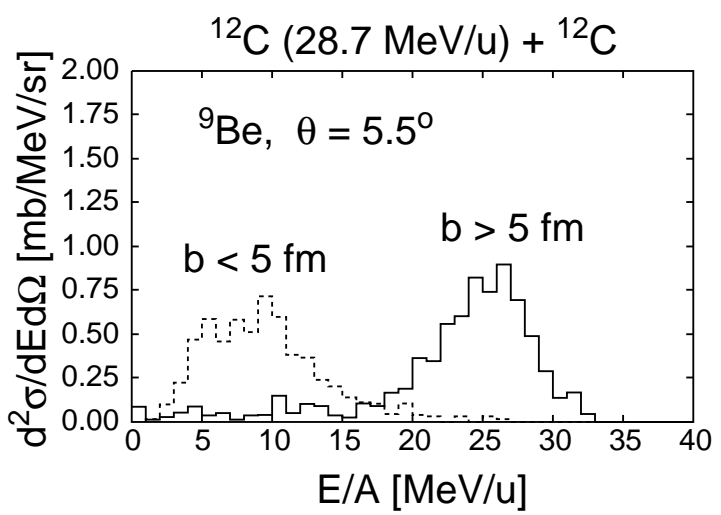

FIG. 6. Calculated contribution to the energy spectrum of ${ }^{9} \mathrm{Be}$ at $\theta=5.5^{\circ}$ from peripheral $(b>5 \mathrm{fm})$ and central $(b \leq 5 \mathrm{fm})$ collisions in the case (c) of stochastic collision process.

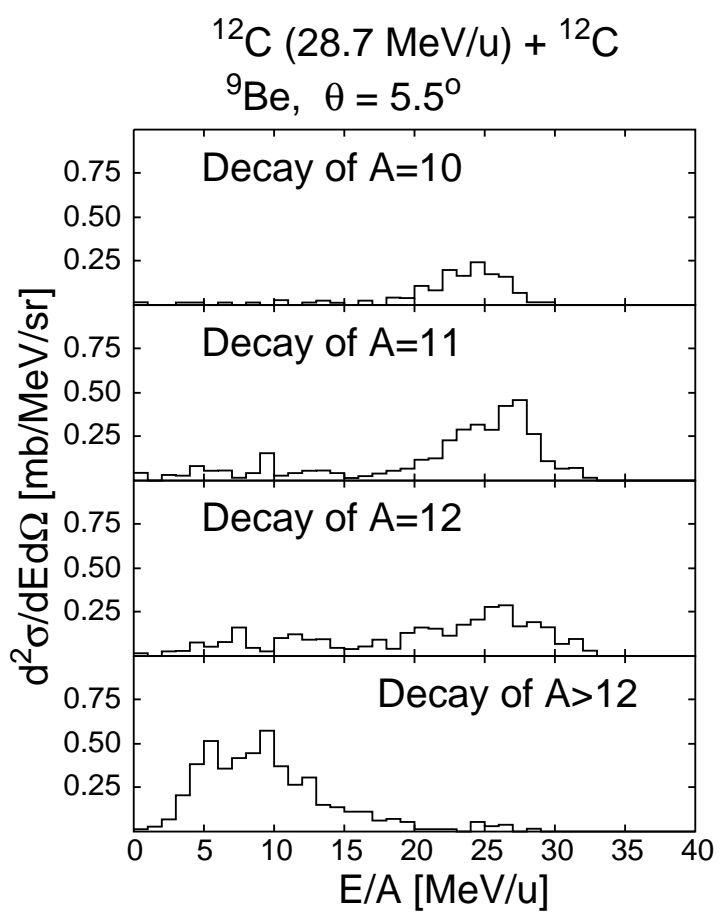

FIG. 7. Contribution to the energy spectrum of ${ }^{9} \mathrm{Be}$ at $\theta=5.5^{\circ}$ from statistical cascade decay of excited fragments, with mass numbers 10, 11, 12 and more, produced in AMD calculation for the case (c) of stochastic collision process. 


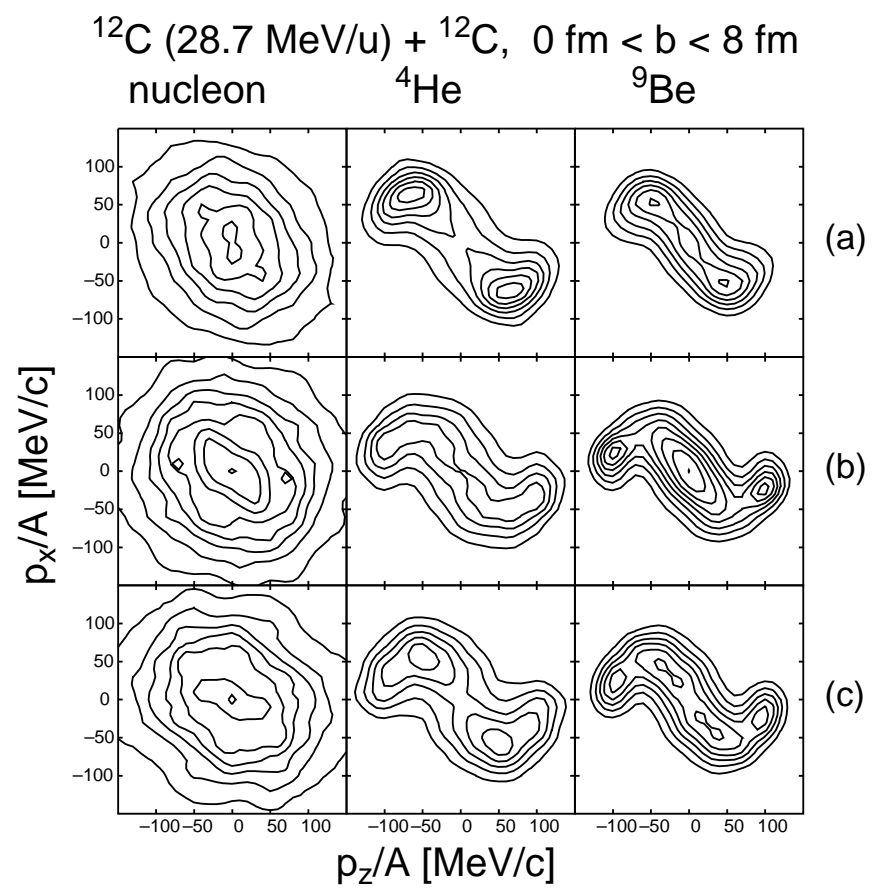

FIG. 8. Contour maps of distribution of momentum component in the reaction plane of nucleons, alpha particles and ${ }^{9} \mathrm{Be}$ fragments for three cases (a), (b) and (c) of stochastic collision process. The $z$-axis is the beam direction and the $z x$-plane is the reaction plane. The distribution has been smoothed out by the Gaussian distribution with the standard deviation $\Delta p / A=10$ $\mathrm{MeV} / c$.

\section{Low energy part of spectrum}

In the energy spectrum of ${ }^{9} \mathrm{Be}$ (Fig. 5), there has appeared another bump in the low energy part in our calculation. This bump has turned out to come from central events with impact parameters smaller than $5 \mathrm{fm}$ (see Fig. 6) and therefore has been produced by completely different reaction mechanism from the projectile fragmentation. From Fig. 7, it is seen that this bump is due to the decay of compound nuclei with mass number larger than 12. The height of this bump is sensitive to the cross section of stochastic collisions as can be seen from Fig. 5. In Fig. 8, calculated distributions of the momentum component in the reaction plane of various fragments are shown as contour maps for three types of stochastic collision process. We can see the projectile fragmentation peak in the cases (b) and (c). In addition to this peak, there has appeared another hill which comes from central events as can be seen by the comparison with Fig. 9 which shows the contribution from impact parameter range $b<5 \mathrm{fm}$. The shape of this hill is dependent on the cross section of stochastic collisions. It is concentrated at the origin in the case (b), while it is separated into two mountains in the cases (c) and (a). This feature is, of course, responsible for the cross section dependence of the low energy part in Fig. 5, and is understandable by the idea that the dissipation of incident kinetic energy of projectile and target in central events is smaller for smaller cross section of stochastic collisions. As long as the central events are 


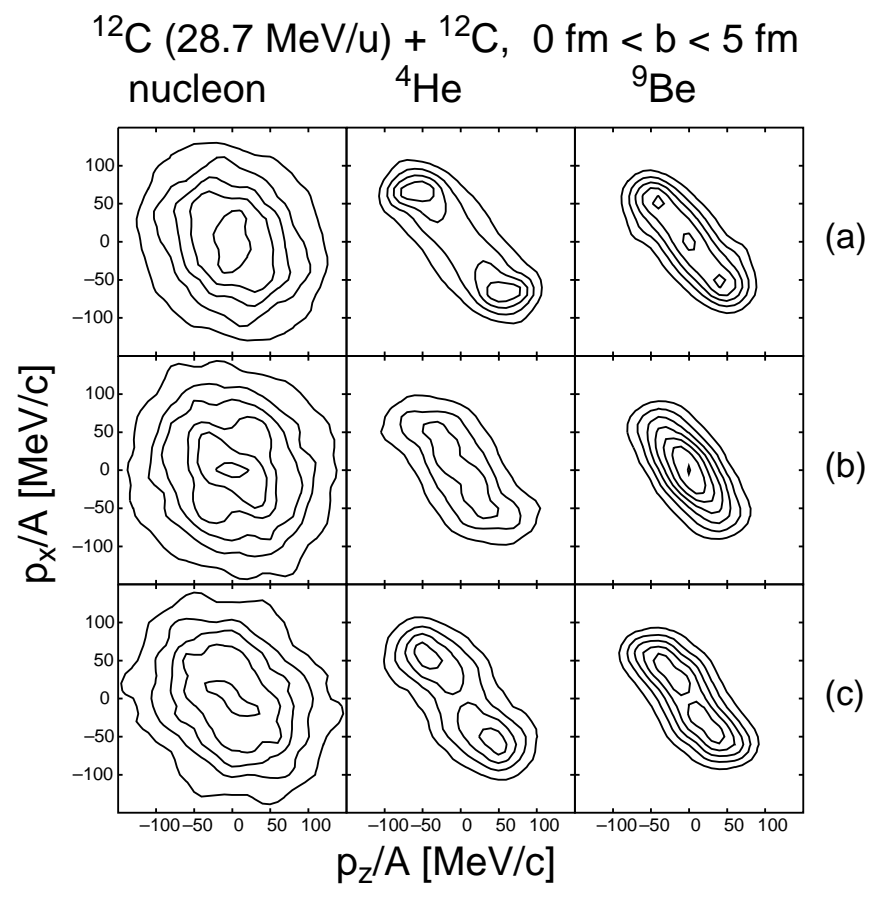

FIG. 9. The same as Fig. 8, but with the contribution only from central collisions $(b<5 \mathrm{fm})$.

concerned, the cases (a) and (c) produce similar results.

We can see similar dependence on stochastic collision process of the shape of the hill in the momentum distribution of alpha particles in Fig. 8 and Fig. 9. This means that the momentum distribution of fragments such as alpha and ${ }^{9} \mathrm{Be}$ is a good indicator of the cross section of stochastic collisions in the reaction ${ }^{12} \mathrm{C}+{ }^{12} \mathrm{C}$, though this situation may depend on the mass number of target since the dissipation of kinetic energy of projectile is expected to be large for heavy target. The momentum distribution of nucleons, on the other hand, does not show clear dependence on the cross section, while the spreading of distribution is larger if nucleon-alpha collisions are included. The last feature is considered to appear because the momentum of nucleon which has been scattered by an alpha cluster is larger than the momentum of nucleon which has been scattered by another nucleon. It is desirable to have data of nucleon energy spectra in this reaction.

\section{E. Discussion on the collective momentum flow}

In the calculated energy spectrum of ${ }^{9} \mathrm{Be}$ at the forward angle (Fig. 5), there has appeared a dip, which does not exist in the data, between the projectile fragmentation peak and the low energy bump. This dip corresponds to the valley in the momentum distribution (Fig. 8) which has appeared as the result of clear separation of two components, i.e., projectile fragmentation peak and the low momentum hill. The direction of the ridge of the hill in the momentum distributions of fragments is about $-45^{\circ}$, which means that the projectile and the target go around each other and are deflected by this angle on an average. This clear 


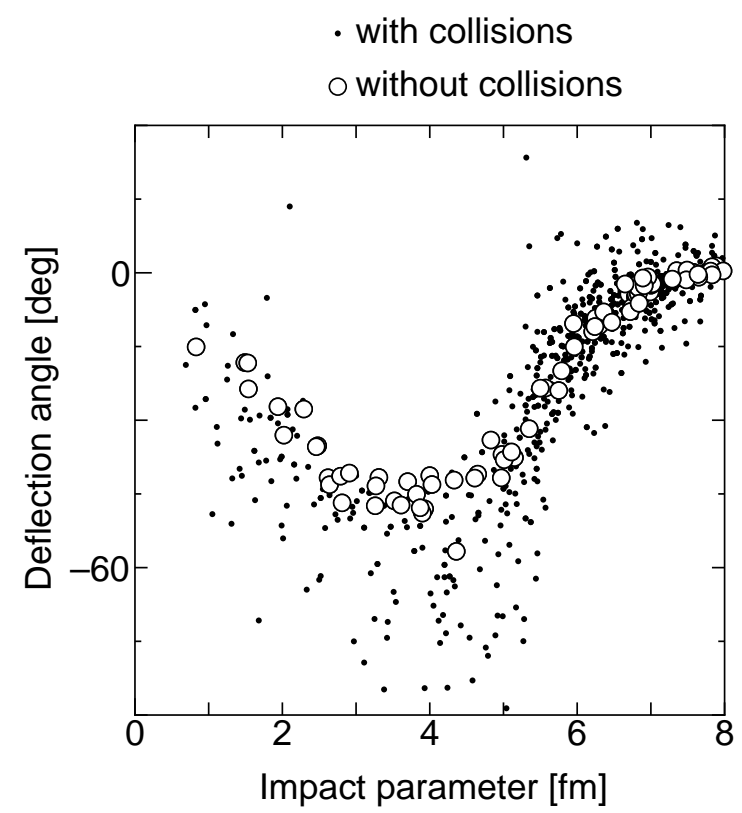

FIG. 10. Deflection angle of the ${ }^{12} \mathrm{C}$ fragments produced in AMD calculation before statistical cascade decay as a function of impact parameter. Open circles show the result of calculation without any stochastic collisions, and dots show the result of the calculation for the case (c) of stochastic collision process.

flow pattern in calculation can be understood from Fig. 10, where the emitted angles of ${ }^{12} \mathrm{C}$ fragments before statistical decay are plotted as a function of impact parameter for two calculations with and without collisions. We can see that the flow angle, about $-45^{\circ}$, has been caused not by the effect of stochastic collisions but by the mean field effect, and the deflection angle suddenly changes from $-10^{\circ}$ to $-45^{\circ}$ when the impact parameter changes from $6 \mathrm{fm}$ to $5 \mathrm{fm}$. If this change were more moderate and/or the negative flow were smaller, the separation between two component in the momentum distribution would not have been so clear and the valley in the energy spectrum of ${ }^{9}$ Be would have been filled up. In fact, we have checked that if the Gogny force [13] is used instead of the Volkov force, the calculation without collisions gives the negative flow angle which is about half of that with the Volkov force. We get the energy spectrum of ${ }^{9} \mathrm{Be}$ shown in Fig. 11 by artificially rotating the momenta of produced fragments by $+20^{\circ}$ in the reaction plane when $\left|p_{z} / A\right|<75 \mathrm{MeV} / c$.

In Fig. 12, calculated energy spectra of alpha particles of three detection angles are compared to the data [14]. The underestimation of the mid-energy part of the spectrum of $6^{\circ}$ and the overestimation of the cross section at $25^{\circ}$ can be again understood as to be due to the above-mentioned fact that the negative flow is too large in the present calculation by the use of the Volkov force as the effective interaction. We have not included alpha-alpha elastic collisions as the residual interaction since our main interest has not been in the spectrum of alpha particles but in the spectra of projectile-like fragments such as ${ }^{9} \mathrm{Be}$. The incorporation of alpha-alpha elastic collisions may be expected to have some effects in enhancing the high energy component of the spectrum of alpha particles. 


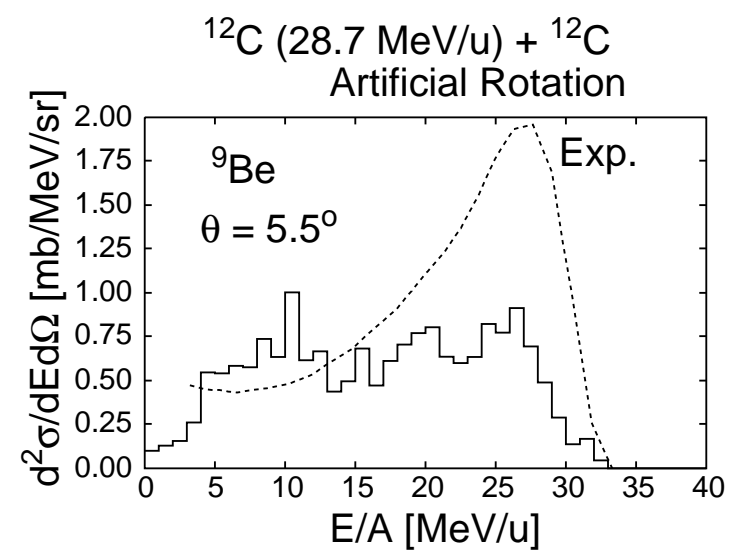

FIG. 11. Energy spectrum of ${ }^{9} \mathrm{Be}$ at $\theta=5.5^{\circ}$ obtained by artificially rotating the momenta by $+20^{\circ}$ in the reaction plane when $\left|p_{z} / A\right|<75 \mathrm{MeV} / c$.

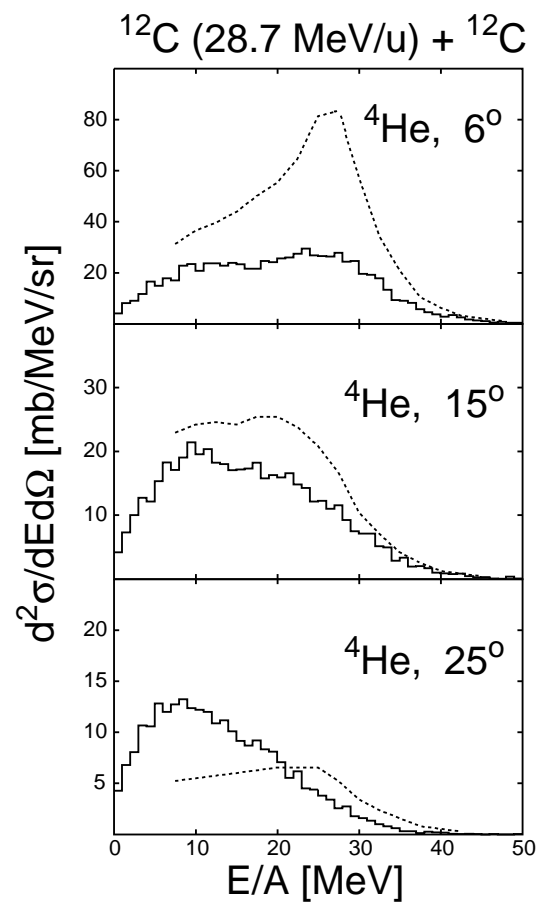

FIG. 12. Energy spectra of alpha particles. Results of calculation are shown by histograms, and the data are shown by dotted curves. Alpha particles produced in the calculation are assumed to have momentum spreading of Gaussian form with the standard deviation $\Delta p / 2$, where $\Delta p$ is the momentum spreading of the wave packet of center-of-mass of alpha particles. 


\section{SUMMARY}

In this paper, we have analyzed the mechanism of the reaction ${ }^{12} \mathrm{C}+{ }^{12} \mathrm{C}$ with the incident energy $28.7 \mathrm{MeV} /$ nucleon with the antisymmetrized version of molecular dynamics (AMD) by paying attention mainly to its dependence on the stochastic collision process. Mass distribution of produced fragments, especially with mass number smaller than 12 , has turned out to be insensitive to the stochastic collision process, and is always well reproduced including the shell effect. The momentum distribution of fragments is, on the other hand, very sensitive to the collision process. With the inclusion of many-body nature expressed representatively by the nucleon-alpha collisions, the observed feature of the projectile fragmentation has been reproduced by calculation in this reaction with relatively low incident energy. The low momentum component in the momentum distribution of fragments such

as alpha particles and ${ }^{9} \mathrm{Be}$, which comes from central events, is sensitive to the value of the cross section of stochastic collisions and hence is expected to give precious information about the cross section of stochastic collisions.

Unfortunately we have not reproduced the data of momentum distribution perfectly since the adopted effective interaction results in too large attraction between the projectile and the target, and therefore the negative momentum flow is too large. This means, in turn, that the close analysis of momentum distribution can give the information about effective interaction, or the momentum dependence and the density dependence of the mean field. Especially the collective momentum flow of fragments such as alpha particles seems to be a very clear quantity which reflects the effective interaction, compared to the flow of nucleons. The analysis of the flow of fragments with other effective interactions, such as Gogny force, is an interesting future problem.

\section{ACKNOWLEDGMENTS}

The computational calculation for this work has been financially supported by Research Center for Nuclear Physics, Osaka University. 


\section{REFERENCES}

[1] J. Aichelin and H. Stöcker, Phys. Lett. B176, 14 (1986); J. Aichelin, Phys. Rep. 202, 233 (1991).

[2] T. Maruyama, A. Ohnishi, and H. Horiuchi, Phys. Rev. C42, 386 (1990).

[3] A. Ono, H. Horiuchi, T. Maruyama and A. Ohnishi, Phys. Rev. Lett. 68, 2898 (1992).

[4] A. Ono, H. Horiuchi, T. Maruyama and A. Ohnishi, Prog. Theor. Phys. 87, 1185 (1992).

[5] H. Feldmeier, Nucl. Phys. A515, 147 (1990).

[6] H. Horiuchi, T. Maruyama, A. Ohnishi, and S. Yamaguchi, in Proc. Int. Conf. on Nuclear and Atomic Clusters, Turku, 1991, edited by M. Brenner, T. Lönnroth and F. B. Malik, (Springer); in Proc. Int. Symp. on Structure and Reactions of Unstable Nuclei, Niigata, 1991, edited by K. Ikeda and Y. Suzuki, (World Scientific).

[7] Y. Kanada, master thesis, Kyoto University, 1993.

[8] A. Volkov, Nucl. Phys. 75, 33 (1965).

[9] A. Houdayer, N. E. Davison, S. A. Elbakr, A. M. Sourkes, W. T. H. van Oers and A. D. Bacher, Phys. Rev. C18, 1985 (1978).

[10] T. Maruyama, A. Ono, A. Ohnishi and H. Horiuchi, Prog. Theor. Phys. 87, 1367 (1992).

[11] F. Pühlhofer, Nucl. Phys. A280, 267 (1977).

[12] J. Czudek, L. Jarczyk, B. Kamys, A. Magiera, R. Siudak, A. Strzałkowski, B. Styczen, J. Hebenstreit, W. Oelert, P. von Rossen, H. Seyfarth, A. Budzanowski, and A. Szczurek, Phys. Rev. C43, 1248 (1991).

[13] J. Dechargé and D. Gogny, Phys. Rev. C43, 1568 (1980).

[14] A. Szczurek, A. Budzanowski, L. Jarczyk, A. Magiera, K. Möhring, R. Siudak and T. Srokowski, Z. Phys. A338, 187 (1991). 